\section{Platform for itinerant maritime diffusion. A national marine education network}

PALABRAS CLAVE • EDUCACIÓN MARINA · ACUARIO INVESTICACIÓN · DIFUSIÓN · BUOUE

KEYWORDS • MARITIME EDUCATION · AOUARIUM RESEARCH · DIFFUSION - VESSEL

\section{RESUMEN}

El proyecto tiene como propósito la creación de una red de investigación marina que incorpore la difusión y educación de las personas como uno de sus principales objetivos. Hoy en día, Chile se enfrenta a una excesiva concentración de los esfuerzos de difusión en la zona central del país, junto a una mala distribución y escasos programas de investigación para las áreas marinas protegidas existentes. La propuesta, entonces, replantea la forma en que se concibe un centro de difusión, aprovechando la necesidad de desplazamiento propia de los cruceros de investigación marina.

La necesidad de un proyecto así surge de manera inherente al analizar la realidad nacional respecto del estado de conservación de nuestro mar, su nive de estudio y la falta de conciencia social sobre su cuidado, considerando que parte de la economía del país se desarrolla a partir de sus riquezas, como la pesca, la navegación y el turismo.

\section{ABSTRACT}

The purpose of the project is to create a national maritime research network, whos main objective is the diffusion and education. Nowadays, Chile faces an enormous concentration of diffusion efforts in the central zone of the country, together with an inefficient distribution and scarce research programs for the existing marine protected areas. Thus, the center is co displacement of marine research cruises.

The need for such a project arises inherently when analyzing the national reality regarding the state of conservation of our sea, its level of study and the lack of social awareness, in regards to its care, considering that part of the country's economy develops from its riches, such as fishing, navigation and tourism.

\title{
Plataforma para la difusión marina itinerante
}

\author{
Red nacional de educación marina*
}

ARQ. FRANCO MARRESE TAYLOR ·Santiago, Chile ·f.marrese.t@gmail.com

\section{DIAGNÓSTICO NACIONAL SOBRE CONSERVACIÓN MARINA: PROBLEMAS DE DISTRIBUCIÓN}

Cuando se habla sobre el territorio chileno, generalmente se hace referencia a la superficie terrestre, correspondiente solo al 16,9\%. El $83,1 \%$ del territorio nacional se compone de superficie marina, que junto a la distribución geográfica del territorio ubica a Chile como el quinto país con mayor longitud costera del mundo, alcanzando los $83.850 \mathrm{~km}$ de largo (SUBPESCA, 2005).

El mecanismo mediante el cual se protegen los ecosistemas acuáticos son las áreas marinas protegidas (AMPs). Chile solo posee 17, y su superficie se concentra en las islas oceánicas. Esto deja cerca del $1 \%$ de la costa protegida, evidenciando un problema de distribución agravado por la ausencia de planes administrativos y programas de investigación (Sierralta, Serrano, Rovira y Cortés, 2011).
Pero la protección no recae exclusivamente en las autoridades, sino también en las actividades humanas que se desarrollan en el mar, cuya intensidad se ha convertido en una de las principales amenazas. A pesar de la importancia de la creación de conciencia para incentivar a la población a involucrarse (Rovira, 2007) (Squella, 2001), Chile no cuenta con un plan nacional de educación marina (Economía y Negocios, 2013), y posee solo cinco centros de difusión, todos de escala pequeña y concentrados en las regiones Metropolitana y de Valparaíso (FIGURA 1).

Por otra parte, existen solo siete centros de investigación, los que no logran abarcar la longitud ni la superficie de la costa, además de no poseer la infraestructura y el equipamiento suficiente. Actualmente la Armada de Chile cuenta con una única unidad de transporte marino para la realización de cruceros de investigación. Si se compara esta cifra con la extensión costera entre diferentes países, Chile está muy por debajo de la norma (CONA, 2005) (FIGURA 2).

* Artículo realizado a partir de los resultados del proyecto de título "Plataforma para la difusión e investigación marina itinerante: red de educación e investigación marina" presentado por el autor en enero de 2018 en la Facultad de Arquitectura y Urbanismo de la Universidad de Chile para obtener el título de Arquitecto (Profesor guía: Manuel Amaya Díaz). 
1. Diagnóstico nacional: protección, investigación y difusión. Elaboración propia, 2017.

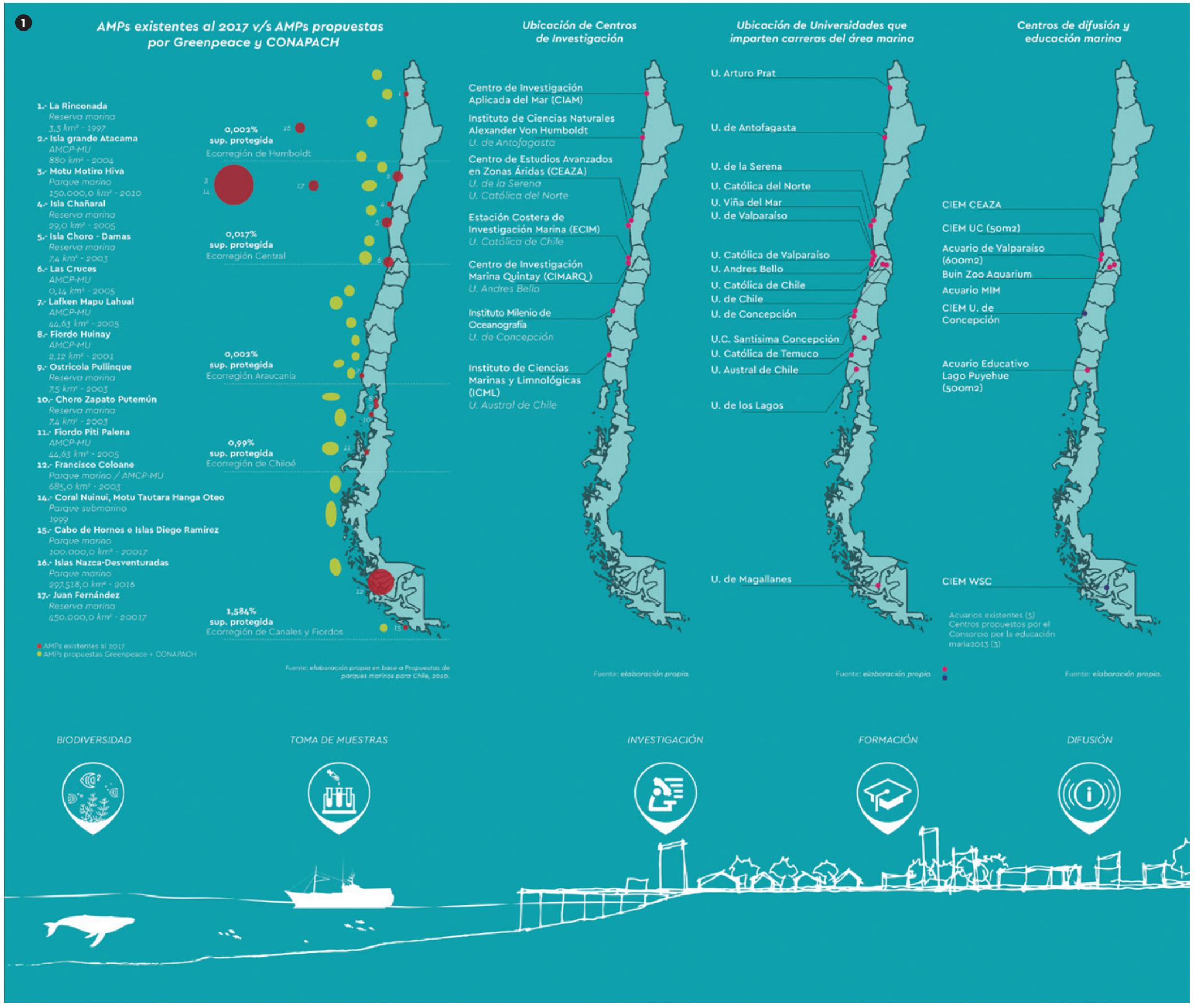


2. Escenario mundial: cantidad de buques de investigación según longitud de costa por país. Elaboración propia, 2017.
3. Propuesta conceptual y planteamiento a escala marítima: definición de estaciones terminales. Elaboración propia, 2017.
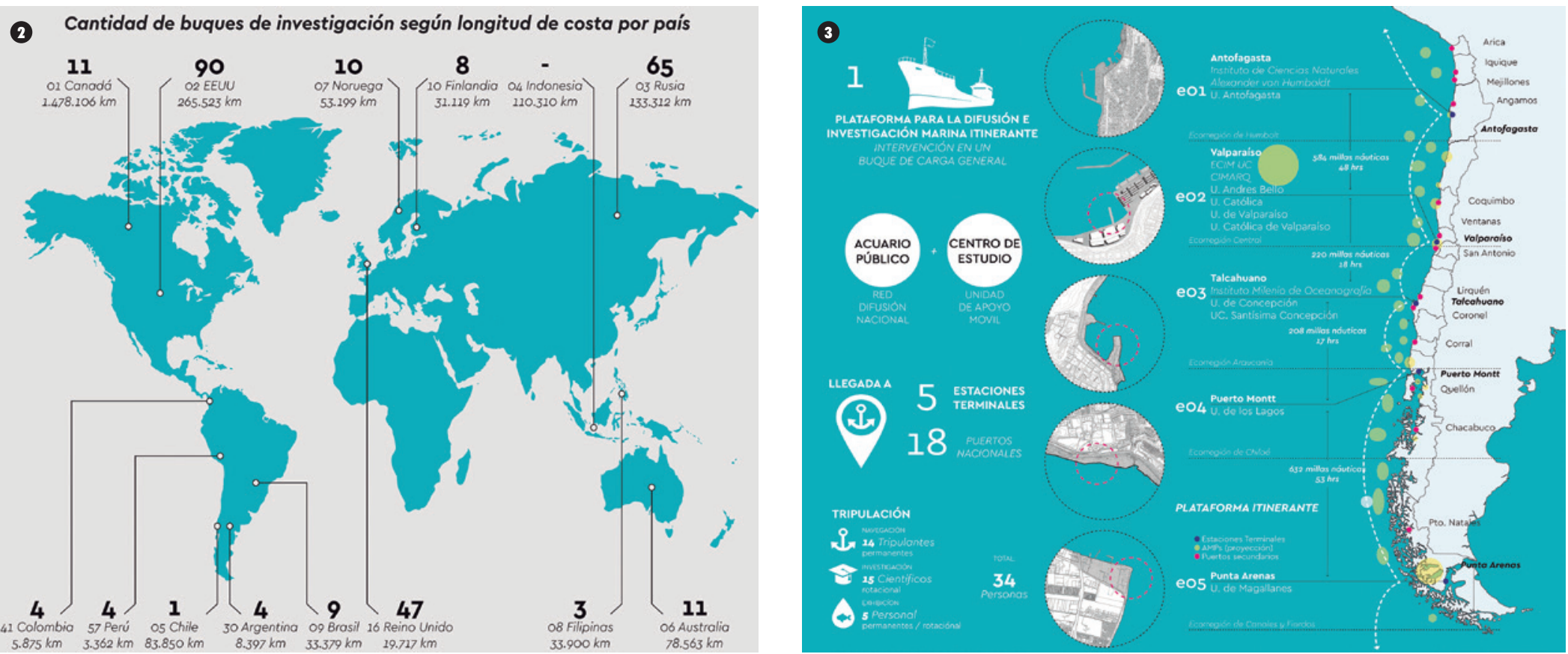

\section{ESCALA MARITIMA: RED NACIONAL DE EDUCACIÓN MARINA}

La configuración geográfica terrestre de Chile dificulta el estudio de las AMPs y la llegada del conocimiento a todos los sectores, concentrando los esfuerzos en las zonas más pobladas y no necesariamente más vulnerables. La posibilidad de implementar infraestructura en cada región para monitorear e investigar, además de desarrollar centros de difusión, conlleva un extenso período de tiempo y un alto nivel de inversión, lo que dilata una situación de carácter perentorio. Cabe preguntarse żcuál es la mejor estrategia para abordar las conservación marina, dada nuestra condición geográfica nacional? ¿̇cómo se puede aportar al desarrollo de la educación y estudio marino desde la arquitectura?
La propuesta toma como elemento central la necesidad de desplazamiento de los cruceros de investigación marina, los cuales se materializan mediante embarcaciones diseñadas para albergar la infraestructura científica permitiendo el estudio de zonas remotas. A partir de esto se plantea una plataforma itinerante capaz de desplazarse a lo largo de la costa, integrado las aristas de estudio y difusión en un mismo espacio, con el fin de ampliar la cantidad de zonas donde se podrá realizar investigación científica al mismo tiempo que se logrará descentralizar los esfuerzos de educación (FIgURA 3).

Se plantea así una red de estaciones terminales cuyo motor principal corresponde a la plataforma itinerante, materializada mediante la intervención de un buque de carga general, transformando su expresión formal y espacialidad interior. Se incorpora el programa base de un crucero de investigación (laboratorios, salas de estudio y equipos) junto a espacios de exhibición, configurando un acuario flotante capaz de recorrer la costa nacional. Cuando la plataforma está navegando, se realizarán expediciones de estudio, mientras que al encontrarse atracada, esta abrirá sus puertas al público a modo de un centro de educación y difusión marina. Se plantean cinco estaciones terminales, una dentro de cada una de las cinco ecorregiones marinas definidas por SullivanSealy y Bustamente (Rovira et al., 2008), que permitan el acceso y la integración del elemento marítimo al contexto terrestre. 
4. Estación terminal Puerto Montt: estrategias urbanas. Elaboración propia, 2017.

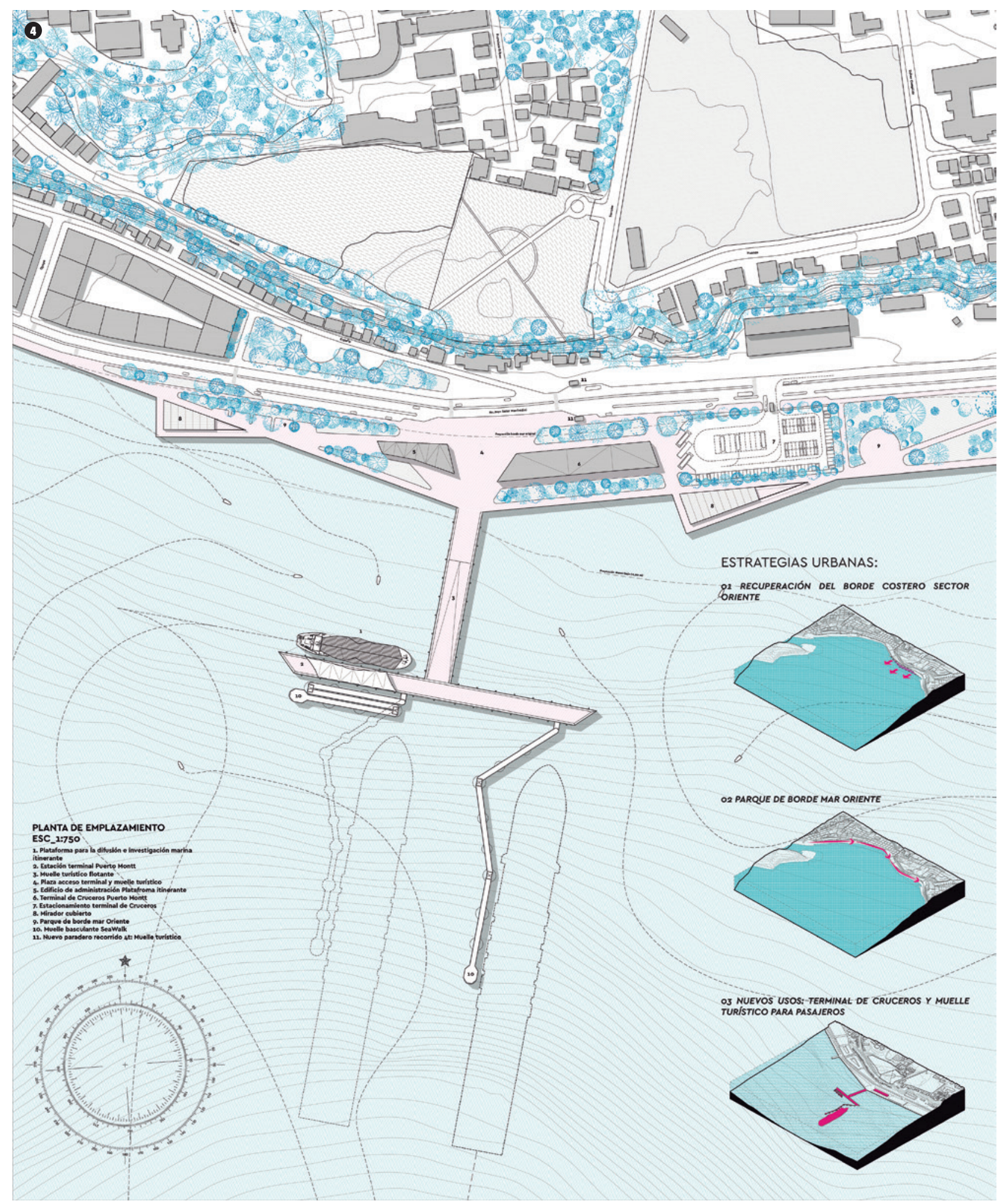


5. Reinterpretación del buque: gran ventana hacia el mundo submarino. Elaboración propia, 2017

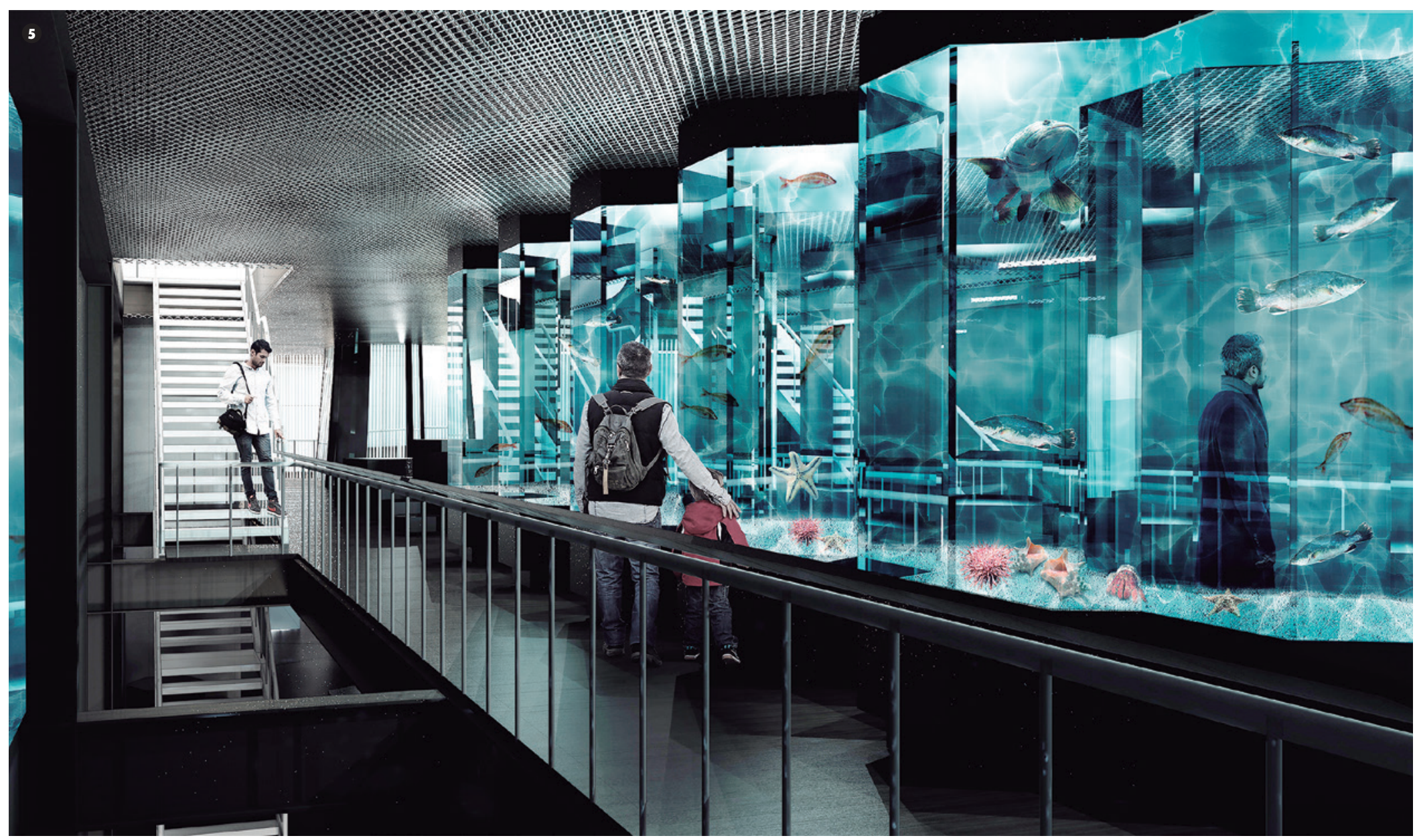

\section{ESCALA ARQUITECTÓNICA: PLATAFORMA ITINERANTE Y ESTACIÓN PUERTO MONTT}

Acorde a la iniciativa del municipio de Puerto Montt de desarrollar un terminal exclusivo para pasajeros en el sector de caleta Pichi Pelluco, se propone incorporar a esta la propuesta de Estación Terminal para la Plataforma, permitiendo así mayor diversidad de usos para el espacio, reactivando una zona que actualmente se encuentra desvalorizada. Para ello se recupera el borde costero desplazando la línea de costa por sobre la zona de intermarea, extendiendo el parque de borde mar proveniente de caleta Angelmó. Se ubican así el terminal de cruceros y el edificio de apoyo logístico a la plataforma en el sector, configurando un espacio de acceso a los muelles basculantes que permitirán el atraque de los cruceros. Por último, se cubre uno de los muelles con una membrana tensada, generando un espacio flexible que permita configurar el espacio de acceso a la plataforma con programas de apoyo (FIGURA 4).

La intervención sobre la embarcación nace a partir de la interpretación metafórica del buque como una gran ventana hacia el mundo submarino que, a la inversa de las experiencias tradicionales de centros de difusión, traslada al visitante a una plataforma flotante distinta de la terrestre, logrando que este se sumeria en el desconocido mundo marino (FIGURA 5). Debido a que gran parte del proyecto queda sumergido, como expresión formal se buscó escapar de la horizontalidad del mar, irrumpiendo la superficie de la misma manera que lo hacen las olas: mediante un movimiento ondulatorio que refleja el movimiento. Así, los diferentes espacios interiores se expresan por sobre el horizonte a través de una cubierta que refleja el impacto de estos en su forma y movimiento. 
6. Sección longitudinal por eje de quilla. Elaboración propia, 2017.

7. Plantas de cubierta de entrepuente (nivel -1) y doble fondo (-2). Elaboración propia, 2017

6

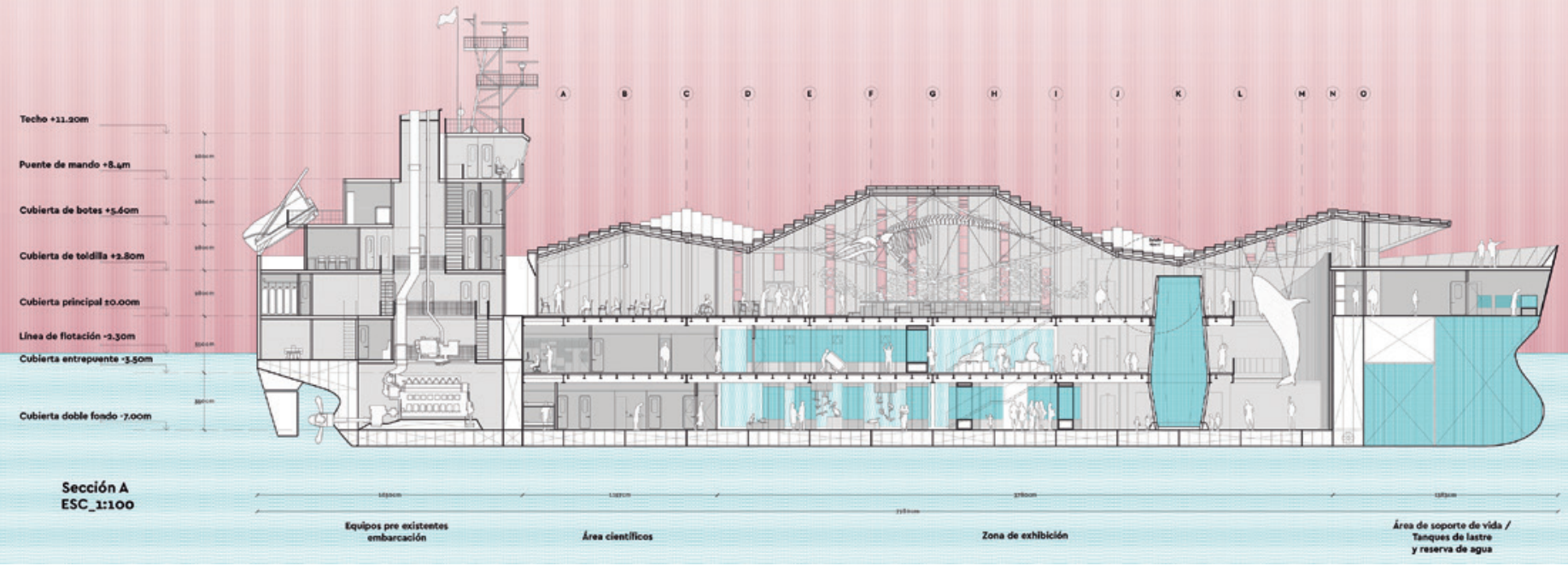

(7)

PLANTA CUBIERTA DOBLE FONDO Nivel-7.00m

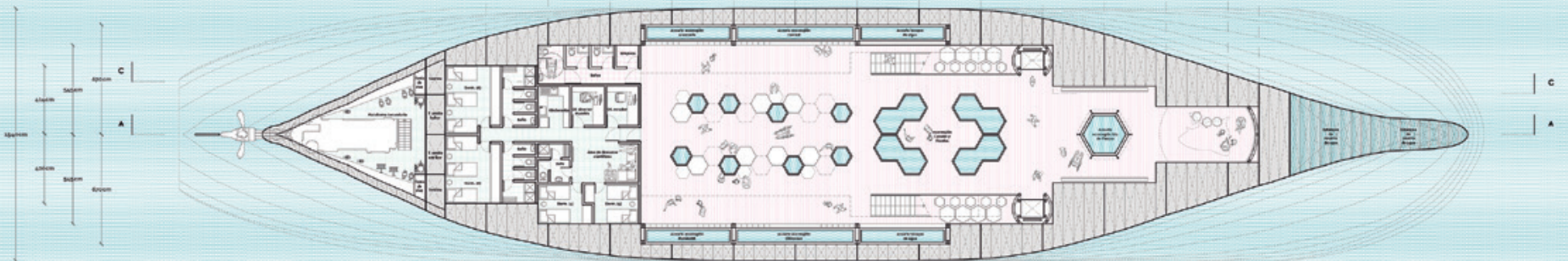

\section{PLANTA CUBIERTA ENTREPUENTE}

Nivel - -3,50m
ESC_L1:200

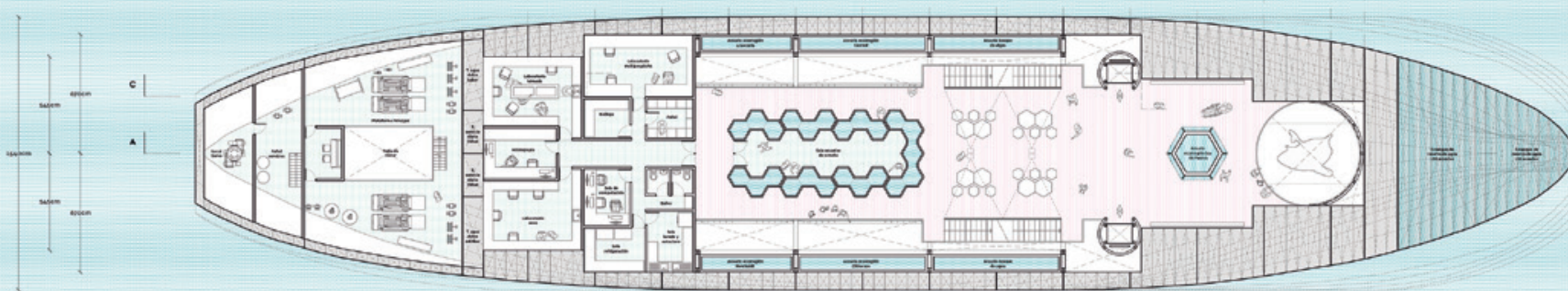


8. Planta de cubierta principal (nivel de acceso). Elaboración propia, 2017.

9. Vista general de la Plataforma ltinerante. Elaboración propia, 2017.

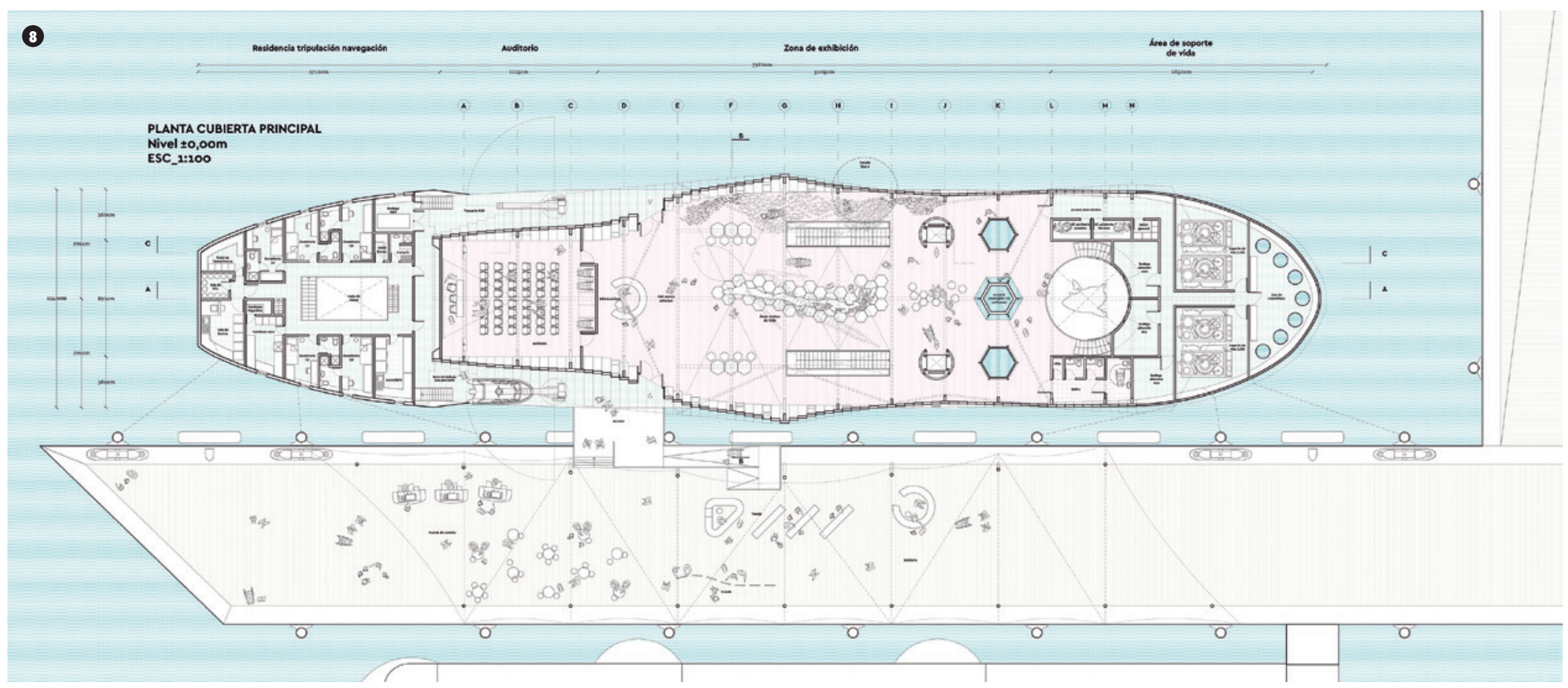

๑

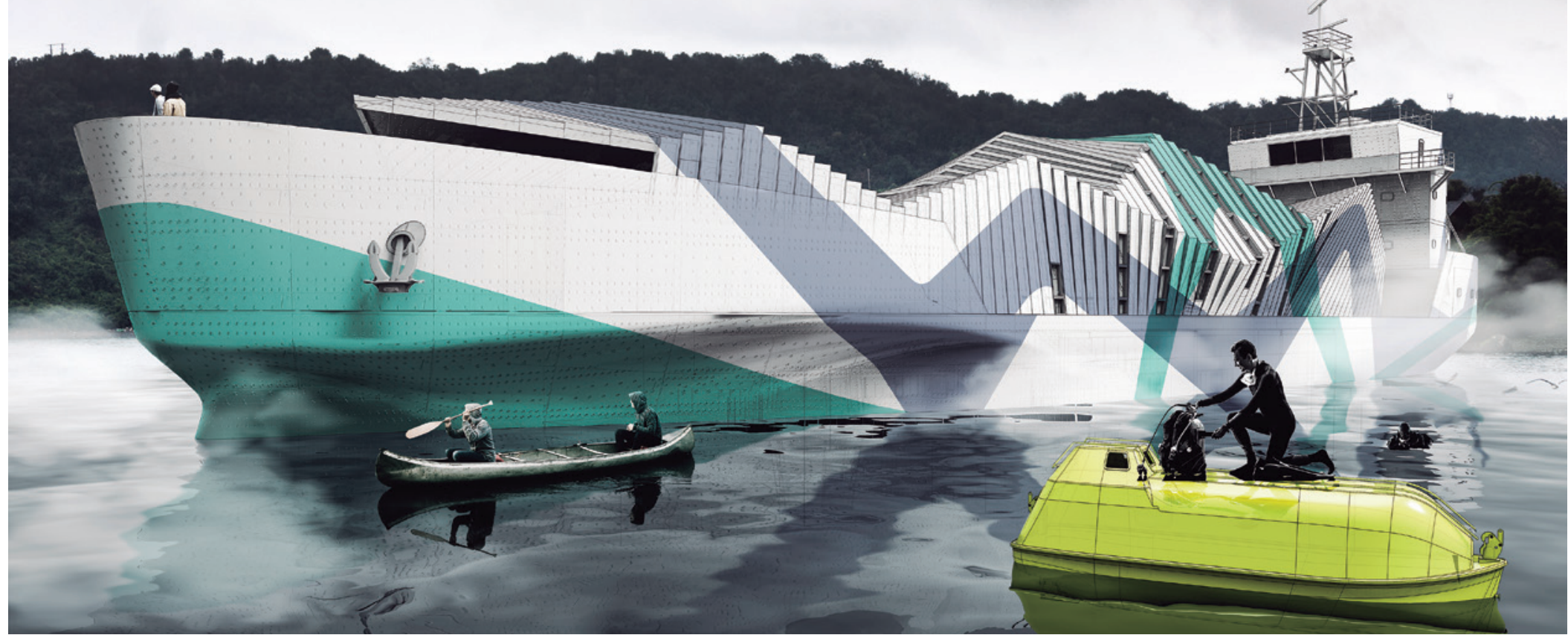


10. Vista interior de la cubierta principal: espacio de introducción al mundo marino nacional. Elaboración propia, 2017.

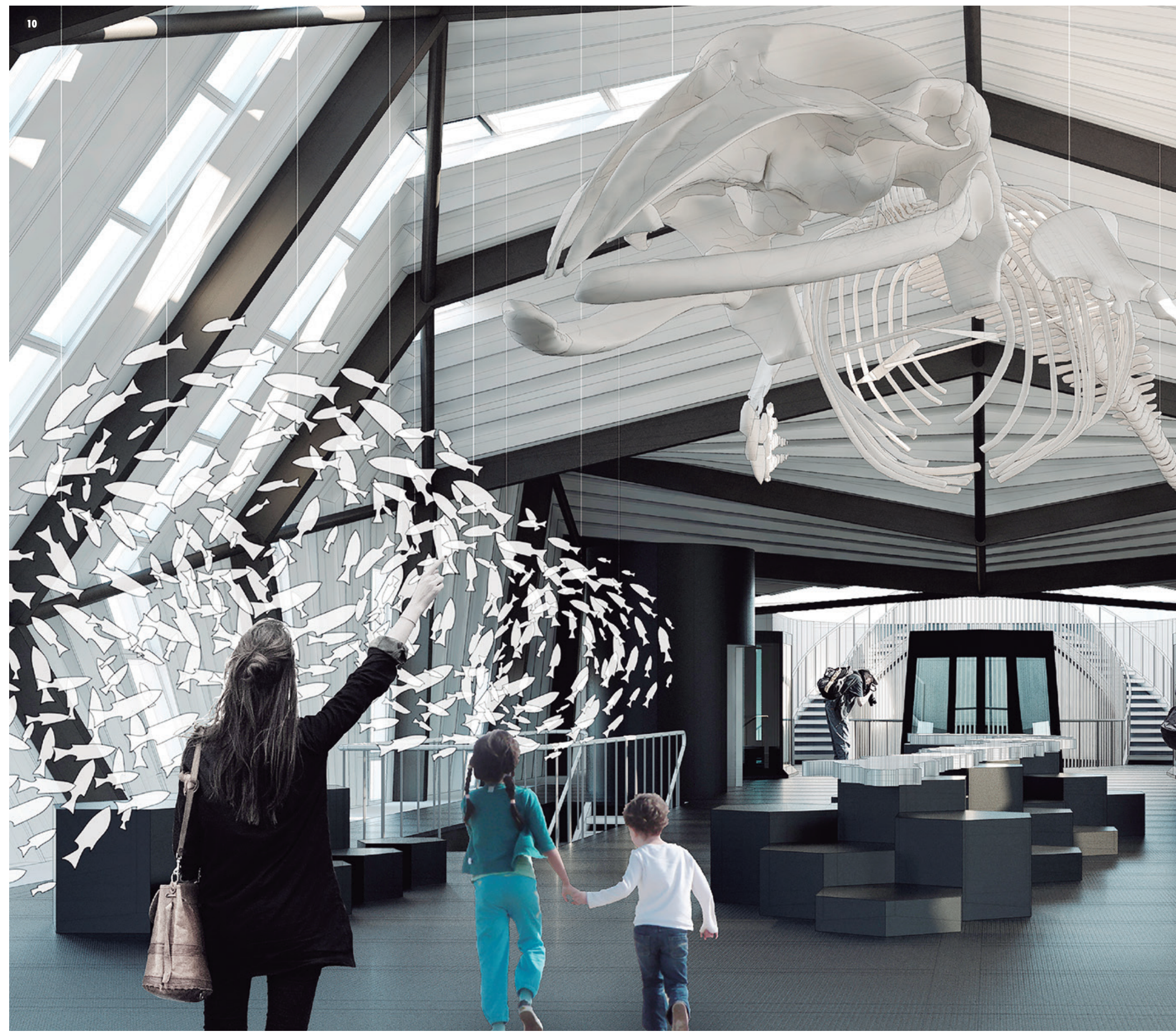




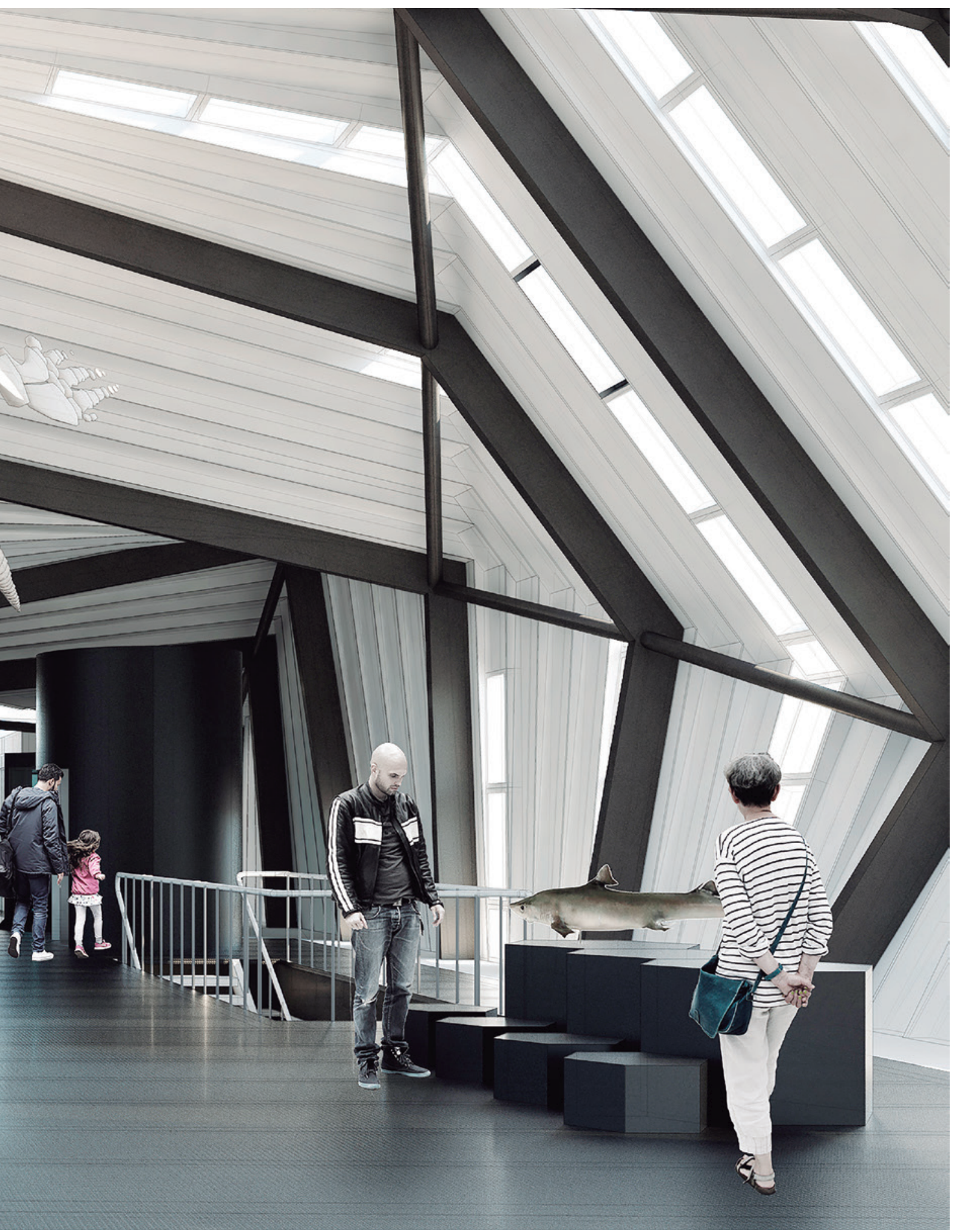

\section{REFERENCIAS BIBLIOGRÁFICAS}

Comité Oceanográfico Nacional, CONA. (2005). Programa CIMAR. Memoria Gestión 19952004. Santiago de Chile: Servicio Hidrográfico y Oceanográfico de la Armada. Recuperado de https://www.yumpu.com/es/document/ view/14109825/programa-cimar-m-e-m-o-r-i-agestion-1995-2004-comite-/66

Economía y Negocios (abril, 2013). La cruzada de Juan Carlos Castilla, el científico que dice que "Chile es largo y ancho" [noticia]. Recuperado de http://www.economiaynegocios.cl/noticias/ noticias. asp? $\mathrm{id}=107822$

Rovira, J. (2007). Informe y diagnóstico de la basura marina en Chile. Recuperado de https://archive. org/stream/BasuraMarinaEnChile/5.Chile-J. Rovira-Final divu.txt

Rovira, J., Ugalde, J., y Stutzin, M. (2008). Biodiversidad de Chile. Patrimonio y desafíos. Recuperado de http://www.uss.cl/biblioteca/ wp-content/uploads/2016/09/Biodiversidad-deChile.-Patrimonio-y-desaf\%C3\%ADos-CONAMA. pdf

Sierralta, L., Serrano, R., Rovira, J., y Cortés, C. (2011). Las áreas protegidas de Chile. Antecedentes, institucionalidad, estadísticas y desafíos. División de Recursos Naturales Renovables y Biodiversidad-Ministerio del Medio Ambiente. Recuperado de http://bibliotecadigital. ciren.cl/bitstream/handle/123456789/6990/ HUM2-0008. pdf? sequence $=1$

Squella, M. (2001). Environmental education to environmental sustainability. Educational Philosophy and Theory, 33(2), 217-230. https:// doi.org/10.1111/j.1469-5812.2001.tb00264.x Subsecretaría de Pesca, SUBPESCA. (2005). Costa de Chile: Zonas de interés pesquero y biológico. Recuperado de http://www.subpesca.cl/ portal//618/w3-article-80144.html 